\title{
REVISIÓN
}

\section{Efecto del consumo de soja en relación con los síntomas de la menopausia}

\author{
Andrea Pérez Rovira ${ }^{a, *}$ y Núria Mach Casellas ${ }^{a, b}$ \\ ${ }^{a}$ Àrea de Cièncias de la Salut, Institut Internacional de Postgrau, Universitat Oberta de Catalunya (UOC), Barcelona, \\ España \\ 'INRA, Département de Génétique Animale et Biologie Intégrative, Jouy-en-Josas, Francia
}

Recibido el 31 de agosto de 2011; aceptado el 14 de marzo de 2012

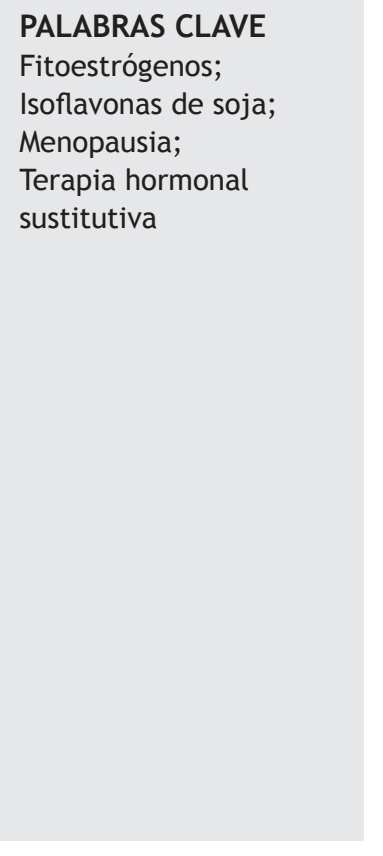

\begin{abstract}
Resumen
La menopausia se caracteriza por el cese de la función ovárica y la disminución hormonal de estrógenos. Estos cambios conllevan una serie de trastornos que pueden afectar a la vida de la mujer. La medicina, debido a la influencia de la industria farmacéutica, tiende a actuar de forma agresiva, y como consecuencia tenemos una población polimedicada. Los médicos suelen recetar tratamientos, como la terapia hormonal sustitutiva (THS), que ayuda a controlar estos síntomas. No obstante, recientemente varios estudios han demostrado efectos adversos asociados a la THS. Actualmente se conoce la influencia de la dieta en muchas de las enfermedades que afectan a la población de los países occidentales. Así, sla soja dietética y algunos suplementos de isoflavonas se han propuesto como alternativas para paliar los síntomas de la menopausia. Varios estudios indican que se puede utilizar las isoflavonas, de gran potencia estrogénica, como alternativa a la THS por su capacidad de reducir los síntomas menopáusicos. No obstante, el conocimiento sobre los efectos de la ingesta de isoflavonas en los síntomas de la menopausia es escaso y las publicaciones científicas recientes muestran resultados dispares. Para esclarecer esta información, el objetivo de esta revisión es describir algunos de los mecanismos mediante los cuales ejercen su acción las isoflavonas, y sus acciones beneficiosas, postulando que, como suplementos alimentarios, se podría utilizarlos como tratamiento complementario para algunos de los síntomas de la menopausia, lo que permitiría disminuir las dosis de THS.

(c) 2011 Asociación Española de Dietistas-Nutricionistas. Publicado por Elsevier España, S.L. Todos los derechos reservados.
\end{abstract}

\footnotetext{
${ }^{*}$ Autor para correspondencia.

Correo electrónico: andrea_provira@hotmail.com (A. Pérez).
} 


\section{KEYWORDS}

Hormone replacement

therapy;

Menopause;

Phytoestrogens;

Soy isoflavones

\section{Introducción}

La menopausia es un proceso fisiológico que se caracteriza por el cese de la función ovárica y la disminución de estrógenos, específicamente el estradiol. La menopausia se reconoce después de 12 meses consecutivos de ausencia de menstruación no relacionada con causas fisiológicas, y aparece alrededor de los 50 años de edad ${ }^{1,2}$.

Los síntomas que aparecen en la menopausia son causados por el déficit estrogénico y de progesteron $a^{2}$ y abarcan: ritmo cardiaco acelerado, sofocos, sudores fríos o insomnio. Asimismo pueden aparecer otros síntomas como: disminución del interés sexual, pérdida de memoria, dolores de cabeza, cambios en el estado de ánimo (como irritabilidad o depresión) ${ }^{3}$, sequedad vaginal, dolores articulares, incontinencia urinaria e infecciones de orina ${ }^{1,2,4}$. A largo plazo, la falta de dichas hormonas puede conducir a una disminución de la masa ósea, lo que comporta un aumento del riesgo de osteoporosis, fragilidad ósea y, en consecuencia, fracturas de huesos en edades avanzadas ${ }^{5}$. En los primeros 5 años tras el cese de la función ovárica se produce la mayor pérdida de masa ósea ${ }^{6}$. Asimismo, con la modificación de las concentraciones hormonales, hay predisposición a modificaciones de la distribución de la grasa corporal, que se deposita preferentemente en la zona abdominal. Estos hechos no son únicamente estéticos, sino que el aumento de peso y la distribución central de la grasa están considerados factores que aumentan el riesgo de padecer enfermedades cardiovasculares ${ }^{7-9}$, diabetes mellitus tipo $2^{10} \mathrm{y}$ algunos tipos de cáncer ${ }^{11-13}$.

Cuando una mujer llega a la menopausia, se le recomiendan ciertos hábitos de vida que harán que esta transición sea más cómoda y llevadera y menos perjudicial para su salud. Evitar el sobrepeso, llevar una dieta saludable, aportar una ingesta adecuada de calcio y vitamina $D$ a través de la dieta, practicar ejercicio físico de manera regular y evitar el tabaco y el alcohol son, entre otras, las principales recomendaciones no farmacológicas en el período perimenopáusico y posmenopáusico.

Debido a que la mayoría de los síntomas están relacionados con la reducción de estrógenos, actualmente la terapia médica de elección para paliar dichos síntomas es la terapia hormonal sustitutiva (THS) basada en estrógenos o derivados (el estrógeno natural más activo y conocido es el estradiol) $)^{3,14,15}$. El objetivo principal de la THS es conseguir concentraciones de estradiol parecidas a las de la premenopausia para aliviar los sofocos, las sudoraciones, las molestias genitourinarias y los cambios emocionales ${ }^{14}$. Además, la THS puede disminuir la pérdida de masa ósea ${ }^{16}$. Con base en estudios observacionales, se concluye que si la THS se inicia poco después de la menopausia y se mantiene durante 5-10 años, se obtiene una reducción del riesgo de fracturas. Sin embargo, dicho beneficio no se observa si se interrumpe el tratamiento ${ }^{6,17}$. Es importante destacar que todas las mujeres a las que no se haya extirpado el útero y que hayan recibido THS a base de estrógenos deberán recibir progesterona, ya que tiene un efecto muy importante en prevenir la aparición de lesiones del endometrio y algunos tipos de cán$\mathrm{cer}^{15}$. Pese a que la THS con estrógenos es la terapia de elección para tratar los síntomas de la menopausia y sus futuras consecuencias, existen algunas contraindicaciones al tratamiento e importantes efectos secundarios que se debe considerar ${ }^{18-21}$

En este sentido, las isoflavonas y los fitoestrógenos derivados de la soja, de los que se ha observado que son compuestos con gran semejanza química a los estrógenos naturales, podrían considerarse como una opción alternativa ${ }^{22} 0$ sinérgica con menor probabilidad de efectos adversos que la $\mathrm{THS}^{23}$. 
Pese a que en modelos celulares y animales, y desde el punto de vista mecanístico, los fitoestrógenos podrían tener efectos similares a los de los estrógenos debido a su unión preferencial al receptor beta, su efectividad en humanos aún es controvertida. Así, en el presente artículo se revisan los últimos hallazgos realizados en torno al efecto de la ingesta de isoflavonas de la soja en relación con el control de las principales enfermedades y los síntomas asociados a la menopausia en humanos. Debido a la gran cantidad de estudios publicados sobre este tema y a los resultados y conclusiones contradictorios de algunos de ellos, los autores de la presente revisión cualitativa han creído conveniente utilizar preferentemente los datos obtenidos a partir de la revisión crítica de la literatura científica y de estudios observacionales de gran tamaño muestral.

\section{Isoflavonas de soja y biodisponibilidad en humanos}

La soja y los extractos de sus granos ocupan hoy un lugar destacado en el mercado de productos proteicos de origen vegetal. En su composición destacan nutrientes como proteínas, glúcidos, lípidos, minerales y fibra. Pero además contiene una serie de sustancias llamadas fitoquímicas, con alta bioactividad ${ }^{24}$. Entre las sustancias fitoquímicas de la soja más estudiadas hasta la fecha, destacan los llamados fitoestrógenos, de los cuales las isoflavonas son la familia más conocida (genisteína, daidceína y glicieteína) ${ }^{25}$.

La divulgación de las potenciales propiedades saludables de estos compuestos ha aumentado el interés por el consumo de productos alimenticios con alto contenido en fitoestrógenos e isoflavonas de soja ${ }^{24}$.

Asimismo, las diferencias observadas entre poblaciones occidentales y orientales en relación con los síntomas derivados de la menopausia y su posible vinculación con la ingesta de soja pueden haber sido importantes en el aumento del interés por dicho alimento ${ }^{24}$. La soja es un ingrediente muy presente en la cocina y la dieta de los países orientales. Las principales fuentes de soja para las mujeres japonesas son la cuajada de soja (47\%), seguida de la soja fermentada (30\%) y la pasta de soja (11\%). Estos productos de soja representan aproximadamente el $88 \%$ de las ocho fuentes de isoflavonas. La que contiene más isoflavonas es la harina de soja, con 2,589 mg/g, seguida de la soja fermentada, con $1,273 \mathrm{mg} / \mathrm{g}$, y la bebida de soja, con $0,357 \mathrm{mg} / \mathrm{g}^{26}$.

En España la media de flavonoides totales consumidos al día es de $313,26 \mathrm{mg} /$ día, mientras que el consumo de isoflavonas es $<0,03 \mathrm{mg} /$ día (tabla 1) ${ }^{27,28}$.

En las regiones orientales donde hay alto consumo de derivados de la soja, los estudios epidemiológicos revelan menor incidencia de enfermedades dependientes de hormonas como, por ejemplo, los cánceres de mama y de ovario o la enfermedad coronaria ${ }^{29}$.

Asimismo las mujeres asiáticas presentan menor incidencia de trastornos asociados al climaterio, como los sofo$\cos ^{30}$.

Es importante destacar que en Europa el consumo de fitoestrógenos no llega a superar $1 \mathrm{mg} /$ día, mientras que en la población oriental el consumo se eleva a $60 \mathrm{mg} /$ día. Se- gún algunos autores, son estas cantidades de fitoestrógenos lo que podría generar efectos fisiológicos ${ }^{30-32}$.

Debemos tener en cuenta que hay muchos factores que pueden influir en la biodisponibilidad de las isoflavonas de soja, y esto claramente influye en la actividad biológica de estas. Aunque los factores o las características habituales de la dieta que influyen en la producción de equol no están claros, esta biodisponibilidad aumenta con el tránsito intestinal rápido y disminuye con una dieta rica en fibra; no se han observado diferencias entre las mujeres premenopáusicas y las posmenopáusicas. No hay un consenso sobre cuál es la fuente de isoflavonas que proporciona mayor biodisponibilidad, y los estudios publicados hasta ahora presentan resultados diferentes, aunque sí se ha observado que varía si se administran como alimento sólido o como bebida líqui$\mathrm{da}^{33-35}$.

\section{Estudios que relacionan el consumo de las isoflavonas de soja con diferentes enfermedades y con síntomas de la perimenopausia y la posmenopausia}

Desde 1990 ha habido un interés creciente en torno a las propiedades saludables que podría ofrecer el consumo de soja para los seres humanos ${ }^{24}$. Desde 1995 , se ha publicado especialmente gran cantidad de estudios de intervención y observacionales en torno a los beneficios del consumo de las isoflavonas de la soja para las mujeres perimenopáusicas y posmenopáusicas ${ }^{36}$. Es importante destacar el papel significativo de la toma de isoflavonas en la prevención de problemas cardiovasculares, el control de la DMO y la reducción y el alivio de los síntomas del climaterio.

\section{Isoflavonas de soja y prevención de problemas cardiovasculares}

El papel de los estrógenos en la prevención de la enfermedad cardiovascular sigue siendo controvertido. En un estudio ${ }^{37}$ realizado con 789 participantes, la ingesta diaria de 25-375 mg de isoflavonas de soja durante 2-24 semanas redujo significativamente la presión arterial sistémica (PAs) en comparación con placebo en adultos con presión arterial normal. De este estudio se concluyó que las isoflavonas de soja disminuyeron significativamente la PAs, pero no la presión arterial diastólica (PAD), sin una relación dosisrespuesta.

Otro estudio, que evaluó en mujeres posmenopáusicas el efecto de la suplementación oral con isoflavonas en la función endotelial medida por la dilatación mediada por flujo, demostró que las isoflavonas aumentaron significativamente la dilatación mediada por flujo. Por lo tanto, los suplementos orales de isoflavonas no mejoran la función endotelial en mujeres posmenopáusicas con gran dilatación mediada por flujo, aunque mejoraron significativamente en las mujeres con baja dilatación mediada por flujo ${ }^{38}$.

Recientemente, también se ha estudiado la relación entre la ingesta de isoflavonas y el colesterol. Los fitosteroles tienen una estructura muy similar al colesterol. Esta característica hace que los fitosteroles sean capaces de inhibir la 
absorción intestinal del colesterol, por lo cual tienen un efecto hipocolesterolémico.

Existen muchos trabajos que relacionan los efectos beneficiosos de los fitosteroles con la reducción de las concentraciones séricas de colesterol y, en consecuencia, la reducción del riesgo de padecer enfermedades cardiovasculares. Un metaanálisis que se llevó a cabo para evaluar los efectos precisos de las isoflavonas de soja en el perfil lipídico observó que las isoflavonas de soja disminuyeron significativamente el colesterol sérico total en $0,10 \mathrm{mmol} / \mathrm{l}$ y el colesterol unido a lipoproteínas de baja densidad (CLDL) en $0,13 \mathrm{mmol} / \mathrm{l}$, aunque no se encontraron cambios significativos en el colesterol unido a lipoproteínas de alta densidad (cHDL) y los triglicéridos totales ${ }^{39}$.

Paralelamente, otro metaanálisis con ocho ensayos aleatorizados, realizado para evaluar con mayor precisión los efectos de las isoflavonas en la concentración de cLDL en la sangre, demostró que la concentración en suero de cLDL en sujetos que consumieron una ingesta de proteína de soja de $50 \mathrm{~g} /$ día y de isoflavonas (96 mg/día) disminuyó en $0,15 \mathrm{mmol} / \mathrm{l}$, en comparación con los que consumieron un contenido bajo de isoflavonas (un promedio de $6 \mathrm{mg} /$ día) con la misma ingesta de proteína de soja. Con la misma ingesta de proteína de soja, el consumo elevado de isoflavonas condujo a una disminución significativamente mayor de CLDL sérico que el consumo bajo de isoflavonas, lo que demuestra que las isoflavonas tienen efectos independientes de la cantidad de proteína de soja consumida para reducir la concentración sérica de $\mathrm{CLDL}^{40}$. En cambio, en otro estudio que pretendía observar el efecto de las isoflavonas de soja en las concentraciones de colesterol, se concluyó que el consumo de isoflavonas de soja no está relacionado con cambios en la concentración de $\mathrm{CLDL}$ o $\mathrm{CHDL}^{41}$. Por último, un estudio a doble ciego que incluyó a 66 mujeres ancianas también encontró mejoras en el cHDL después de la ingesta de isoflavonas de soja. Se dividió a las mujeres en tres grupos: el primer grupo recibió $40 \mathrm{~g} /$ día de proteína de leche descremada; el segundo, $40 \mathrm{~g} /$ día de proteína de soja, y el tercer grupo, $40 \mathrm{~g}$ de proteína de soja con isoflavonas de soja extra. En comparación con el grupo de la leche descremada (grupo placebo), ambos grupos de soja mostraron mejoras significativas tanto en el colesterol total como en el $\mathrm{CHDL}^{42}$. Aunque existen ciertas evidencias de que las isoflavonas son el origen de la mejora en el perfil del colesterol, otros componentes de la soja también pueden ser la causa de esos efectos.

No obstante, en octubre de 1999, la US Food and Drug Administration autorizó mencionar en las etiquetas de los alimentos las propiedades saludables asociadas con proteína de soja y la reducción del riesgo de enfermedades coronarias, aunque según la EFSA no existe una relación evidente de causa y efecto entre el consumo de proteínas de soja y la reducción de las concentraciones de $\mathrm{CLDL}^{43}$.

\section{Isoflavonas de soja y la densidad mineral ósea}

Bolaños et $\mathrm{al}^{14}$ (2010) han demostrado que no hay ninguna diferencia estadísticamente significativa entre la THS y la ingesta de isoflavonas en la reducción del riesgo de fractura vertebral debida a la osteoporosis. Ambas intervenciones parecen ser similares para este resultado. En otro estudio demostraron los efectos de la ingesta de isoflavonas de soja en los marcadores de recambio óseo de mujeres posmenopáusicas. Después de la toma diaria de $56 \mathrm{mg}$ de isoflavonas de soja durante un periodo de 10 semanas a 12 meses, la disminución de desoxipiridinolina urinaria (DPD) fue significativa (el 14,1\% de reducción) en comparación con el valor inicial. No obstante, los suplementos de isoflavonas de soja no afectaron a los marcadores de formación ósea de fosfatasa alcalina y osteocalcina. Son necesarios más estudios para abordar los factores relacionados con los efectos observados de las isoflavonas de soja en el DPD y para comprobar los efectos en otros marcadores de recambio óseo ${ }^{45}$.

En cuanto a la asociación entre ingesta de isoflavonas y DMO, un estudio con 1.240 mujeres menopáusicas demostró que la toma diaria de un promedio de 82 (47-150) $\mathrm{mg}$ de isoflavonas de soja durante 6-12 meses aumenta significativamente la DMO de columna lumbar, sin afectar a la DMO en el cuello femoral, las caderas y el trocánter. Aun así, se necesitan más estudios para abordar los factores que afec$\tan$ a las magnitudes de efecto en la columna vertebral y para verificar el efecto en las caderas ${ }^{46}$. Sin embargo, un estudio que evaluó el efecto a largo plazo ( 2 años) de una toma media de $87 \mathrm{mg}$ de isoflavonas de la soja en la DMO de las mujeres no registró diferencias significativas. Las diferencias en los cambios de DMO fueron $4,1 \mathrm{mg} / \mathrm{cm}^{2} /$ año en la columna lumbar, $-1,5 \mathrm{mg} / \mathrm{cm}^{2} /$ año en el cuello femoral y $2,5 \mathrm{mg} / \mathrm{cm}^{2} /$ año en las caderas. Se obtuvieron resultados similares en los análisis de subgrupos por fuentes de isoflavonas (proteína de soja frente a extracto de isoflavonas) y diferencias étnicas (Asia frente a Occidente). Así, ese estudio concluye que un suplemento alimentario con isoflavonas de soja tiene un efecto mínimamente favorable en la DMO de la columna lumbar y las caderas de las mujeres ${ }^{47}$.

Otro estudio observó que la intervención con isoflavonas atenuó significativamente la pérdida ósea de la columna vertebral de mujeres menopáusicas. La DMO de la columna vertebral de los sujetos que consumieron isoflavonas se incrementó significativamente en $20,6 \mathrm{mg} / \mathrm{cm}^{2}$ en comparación con los sujetos que no consumieron las isoflavonas. Estos efectos favorables se vuelven más significativos cuando el consumo de isoflavonas es $>90 \mathrm{mg} /$ día. Además, si el consumo de isoflavonas de soja se alarga durante 6 meses, puede ser suficiente para ejercer efectos beneficiosos en el hueso de las menopáusicas ${ }^{48}$. Otro estudio también confirmó que la ingesta de isoflavonas inhibe significativamente la resorción ósea y estimula la formación de hueso. La concentración de DPD en las pacientes que consumieron isoflavonas se redujo significativamente en comparación con los sujetos que no consumieron isoflavonas. Estos efectos favorables se dan incluso si el consumo es $<90 \mathrm{mg} /$ día de isoflavonas o si la intervención dura menos de 12 semanas ${ }^{49}$.

Aunque hay alguna evidencia epidemiológica de que la soja puede reducir el riesgo de fractura osteoporótica en mujeres, no se sabe si esta reducción del riesgo también se produce en los varones, por lo que se llevó a cabo un estudio en Singapur en el que se observaron asociaciones específicas de sexo entre la soja y el riesgo de fractura de cadera. Se observó una asociación estadísticamente significativa con el consumo de queso de soja, proteína de soja y las 
isoflavonas con el riesgo de fractura de cadera entre las mujeres pero no entre los varones ${ }^{50}$.

Aunque hay evidencia científica sobre los efectos de las isoflavonas en la DMO, el dictamen científico sobre la justificación de declaraciones de propiedades saludables en relación con las isoflavonas de soja y el mantenimiento de la DMO publicado por EFSA concluye que mantener la DMO es beneficioso para la salud de las mujeres posmenopáusicas, pero que la evidencia aportada no es suficiente para establecer una relación de causa y efecto entre el consumo de isoflavonas de la soja y el mantenimiento de la $\mathrm{DMO}^{51}$.

\section{Isoflavonas de soja y prevención o alivio de síntomas del climaterio}

Las isoflavonas han sido propuestas como una opción alternativa o en sinergia con la THS debido a su similitud con los estrógenos endógenos, aunque Bolaños et al (2010) indican que es difícil establecer resultados concluyentes sobre la ingesta de soja y la reducción de la incidencia de los sofocos en mujeres climatéricas, debido a la gran heterogeneidad encontrada en los estudios publicados ${ }^{52,53}$. No obstante, Howes et $a^{54}$ demostraron que el consumo de isoflavonas reduce el número diario de sofocos a las mujeres menopáusicas, y es evidente en las mujeres que sufren gran número de sofocos cada día.

Igualmente, otro estudio que evaluó la eficacia y los efectos de las terapias no hormonales (p. ej., antidepresivos, con clonidina, con extractos de isoflavonas y con otros medicamentos prescritos) para controlar los sofocos en la menopausia, los inhibidores selectivos de la recaptación de serotonina o inhibidores de la recaptación de la noradrenalina, la clonidina y gabapentina proporcionan evidencias de la eficacia; sin embargo, los efectos son menores que los de los estrógenos. Así, estas terapias no hormonales pueden ser muy útiles para mujeres altamente sintomáticas que no pueden tomar estrógenos ${ }^{55}$. En la misma línea, un ensayo clínico realizado en 190 mujeres pertenecientes a nueve comunidades autónomas españolas con un preparado de isoflavonas de soja durante 4 meses halló una disminución significativa del número de sofocos en un $80-82 \%$ de los casos. Además, se observó una mejoría significativa en los trastornos del sueño, nerviosismo, estado de ánimo depresivo y disminución de la libido ${ }^{56}$.

La EFSA, después de distintos estudios y duraciones con dosis de genisteína, concluye que las pruebas aportadas son insuficientes para establecer una relación de causa y efecto entre el consumo de isoflavonas de la soja y la reducción de los síntomas vasomotores asociados con la menopausia ${ }^{57}$.

\section{Isoflavonas de la soja y prevención de algunos tipos de cáncer}

Diversos estudios relacionan el consumo de fitoestrógenos con la disminución del riesgo de cáncer ${ }^{58-71}$. La posible actividad preventiva del consumo de fitoestrógenos frente a diversos cánceres, especialmente el de mama, ha sido revisada por diversos artículos, gracias a la presencia, entre otros compuestos bioactivos, de las isoflavonas. La ingesta de isoflavonas mediante la dieta también ha sido objeto de estudio durante los últimos años. Recientemente, Dong et $\mathrm{al}^{58}$ han demostrado que el consumo de las isoflavonas de soja se asocia con un riesgo significativo de reducción de la incidencia del cáncer de mama en las poblaciones asiáticas, aunque no en las poblaciones occidentales. El análisis estratificado indicó que la menopausia puede ser un modificador del efecto importante en estas asociaciones, aunque no fue posible identificar una relación dosis-respuesta entre el consumo total de isoflavonas y el riesgo de incidencia de cáncer de mama ${ }^{58}$.

Igualmente, Travis et al ${ }^{64}$ demostraron que reduce el riesgo de cáncer de mama. Las mujeres con alto consumo (por encima de la mediana) revelaron una significativa reducción del $18 \%$ del riesgo relativo. Estos hallazgos indican que aproximadamente $10 \mathrm{mg}$ de isoflavonas al día pueden tener efectos beneficiosos duraderos contra el desarrollo de cáncer de mama ${ }^{68}$. Paralelamente, un estudio en Japón demostró una asociación inversa estadísticamente significativa entre la isoflavona genisteína en plasma y el riesgo de este tipo de cáncer; en cambio, no hubo asociación con la daidzeína en plasma ${ }^{69}$. En otro estudio se examinó el papel de la ingesta de isoflavonas de soja y el riesgo de recurrencia de cáncer de mama en estado de receptores hormonales, la menopausia y la terapia con tamoxifeno en mujeres supervivientes a este tipo de cáncer. Entre las mujeres posmenopáusicas tratadas con tamoxifeno, hubo una reducción de aproximadamente el $60 \%$ en la recurrencia del cáncer de mama comparadas con el consumo más alto y más bajo de daidzeína (> 1.453 frente a $<7,7 \mu \mathrm{g} /$ día). Se concluyó que, si las isoflavonas de soja se consumen en cantidades comparables a las de las poblaciones de Asia, pueden reducir el riesgo de recurrencia del cáncer para las mujeres que reciben la terapia con tamoxifeno y, además, no parece interferir con la eficacia de este tratamiento. De todas formas, serían necesarios otros estudios prospectivos antes de hacer recomendaciones respecto a la ingesta de soja para las supervivientes a cáncer de mama ${ }^{70}$. Por todo ello, se cree que una dieta rica en fitoestrógenos de la soja confiere cierta protección contra esa enfermedad ${ }^{72}$. No obstante, otro estudio que evaluó la relación entre el consumo de soja de la dieta y el riesgo de cáncer de mama concluyó que la ingesta dietética de los alimentos de soja o sus componentes parece ser segura para las mujeres sin cáncer de mama; sin embargo, la seguridad de los suplementos con alto contenido de soja o sus componentes es menos clara. Destaca que es importante evaluar correctamente las recomendaciones dietéticas para todas las mujeres que tengan alto riesgo 0 antecedentes de cáncer de mama ${ }^{59}$. Dados los modestos efectos hormonales de las isoflavonas de la soja y el trébol rojo y el riesgo que pueden suponer para el cáncer de mama, en un estudio se evaluaron los efectos de las isoflavonas de los alimentos o suplementos en un biomarcador de riesgo de cáncer de mama: la densidad mamográfica de la mujer. El estudio concluyó que el consumo de las isoflavonas no altera la densidad de la mama en las mujeres posmenopáusicas, pero sí puede causar un pequeño aumento en la densidad de la mama en las mujeres premenopáusicas ${ }^{60}$.

Debido a los muchos estudios que apuntan a que la ingesta de productos de soja puede proteger contra la aparición de cáncer de mama, dada la considerable cantidad de isofla- 
vonas que contienen, se realizó un metaanálisis en el que se observó que el consumo de isoflavonas daba como resultado una disminución del $20 \%$ en el riesgo relativo del cáncer de mama y, por lo tanto, apoya la hipótesis de que la ingesta de alimentos con soja puede estar asociada con un menor riesgo de cáncer de mama debido a las isoflavonas ${ }^{61}$.

\section{Conclusiones}

El futuro de la suplementación con fitoestrógenos se orienta a la mejora de la salud. Hay gran variedad de estudios que demuestran relación entre la ingesta de fitosteroles, especialmente isoflavonas de la soja, y los síntomas de la menopausia. Gran parte de los resultados coinciden en indicar que la ingesta de isoflavonas no solamente reduce el colesterol total, el cLDL y los triglicéridos, sino que aumenta el CHDL, reduce la presión arterial sistólica y el riesgo de infarto cerebral y de miocardio, así como el cáncer de mama. También señalan que el consumo de isoflavonas de soja mejora la función endotelial en las mujeres con bajo grado de dilatación mediada por flujo y tiene efectos beneficiosos en la proteína C reactiva y aumenta la DMO en la columna lumbar. No obstante, otros estudios demuestran resultados contrarios, lo que indicaría que los estudios realizados hasta ahora para establecer la relación entre el consumo de isoflavonas y la prevención o el alivio de los síntomas del climaterio y las enfermedades asociadas son insuficientes. A pesar de que las relaciones causa-efecto entre el consumo de isoflavonas y la prevención o el alivio de los síntomas del climaterio no están todavía bien definidas, la ingesta de isoflavonas a través de la dieta se considera una posible herramienta para revertir o prevenir los afectos adversos del climeterio y, en particular, las enfermedades metabólicas asociadas.

Es prematuro extraer conclusiones definitivas en relación con el uso de isoflavonas como alternativa a los estrógenos de reemplazo hormonal en mujeres posmenopáusicas. Mientras que los beneficios de la THS en estos síntomas están demostrados, no está tan claro que pueda hacer lo mismo el consumo de fitoestrógenos. Los resultados son esperanzadores, pero se requiere de más estudios para extraer datos concluyentes y sustituir así el tratamiento hormonal por el consumo elevado de isoflavonas.

\section{Conflicto de intereses}

Los autores declaran no tener ningún conflicto de intereses

\section{Bibliografía}

1. Atsma F, Bartelin M, Grobbee D, Van der Schouw Y. Postmenopausal status and early menopause as independen risk factors for cardiovascular disease: a meta-analysis. Menopause. 2006;13:265-79.

2. Becerra A. La edad de la menopausia. Madrid: Díaz de Santos; 2003.

3. Birkhäuser M. Depression, menopause and estrogenes: is there a correlation? Maturitas. 2002;41:3-8.
4. Maclennan AH. HRT in difficult circumstances: are there any absolute contraindications? Climateric. 2011;14:409-17.

5. Phillips PJ, Phillipov G. Bone mineral density -frequently asked questions. Aust Fam Physician. 2006;35:341-4.

6. Recker MD, Lappe J, Davies M, Heaney R. Bone remodelling increases substantially in the years after menopause and remains increased in older osteoporosis patients. J Bone Mineral Res. 2004;19:1628-33.

7. Carr M. The emergence of the metabolic sindrome with menopause. Cardiovasc End. 2003;88:2404-11.

8. Strazzullo P, D'Elia L, Cairella G, et al. Excess body weight and incidence of stroke: meta-analysis of prospective studies with 2 million participants. Stroke. 2010;41:418-26.

9. Schenck-Gustafsson $\mathrm{K}$, Brincat $M$, Erel $C T$, et al. EMAS position statement: Managing the menopause in the context of coronary heart disease. Maturitas. 2011;68:94-7.

10. Vazquez G, Duval S, Jacobs DR Jr, Silventoinen K. Comparison of body mass index, waist circumference, and waist/hip ratio in predicting incident diabetes: a meta-analysis. Epidemiol Rev. 2007;29:115-28.

11. Harvie M, Hooper L, Howell AH. Central obesity and breast cancer risk: a systematic review. Obes Rev. 2003;4:157-73.

12. Moghaddam AA, Woodward M, Huxley R. Obesity and risk of colorectal cancer: a meta-analysis of 31 studies with 70,000 events. Cancer Epidemiol Biomarkers Prev. 2007;16:2533-47.

13. Maclnnis RJ, English DR. Body size and composition and prostate cancer risk: systematic review and meta-regression analysis. Cancer Causes Control. 2006;17:989-1003.

14. López A, Costa i Pagés J. Tractament de la simptomatologia menopáusica. Butlletí Inf Terap. 2007;19:47-52.

15. North American Menopause Society. Estrogen and progestogen use in postmenopausal women: 2010 position statement of The North American Menopause Society. Menopause. 2010;17:24255.

16. MacLean C, Newberry S, Maglione M, et al. Systematic review: comparative effectiveness of treatments to prevent fractures in men and women with low bone density or osteoporosis. Ann Intern Med. 2008;148:197-213.

17. Alberti R, Altimiras J, Bosch M, Camós F, Catalán A. Tractament hormonal substitutiu. Butlletí Inform Terap. 2001;13:1-4.

18. Farquhar C, Marjoribanks J, Lethaby A, et al. Long term hormone therapy for perimenopausal and postmenopausal women. Cochrane Database Syst Rev. 2009;(2):CD004143.

19. Olié V, Canonico M, Scarabin PY. Risk of venous thrombosis with oral versus transdermal estrogen therapy among postmenopausal women. Curr Opin Hematol. 2010;17:457-63.

20. Greiser CM, Greiser EM, Dören M. Menopausal hormone therapy and risk of lung cancer -Systematic review and meta-analysis. Maturitas. 2010;65:198-204.

21. Furness S, Roberts H, Marjoribanks J, et al. Hormone therapy in postmenopausal women and risk of endometrial hyperplasia. Cochrane Database Syst Rev. 2009;(2):CD000402.

22. López Luengo T. Fitoestrógenos, eficacia y seguridad. Ámbito Farmacéutico. 2010;29:86-90.

23. Tempfer CB, Froese G, Heinze G, Bentz EK, Hefler LA, Huber JC. Side effects of phytoestrogens: a meta-analysis of randomized trials. Am J Med. 2009;122:939-46.

24. Messina M. A brief historical overview of the past two decades of soy and isoflavone research. J Nutr. 2010;140: S1350-4.

25. Lemos ML. Effects of soy phytoestrogens genistein and daidzein on brest cáncer growth. Ann Pharmacother. 2001;35: 118-21.

26. Somekawa $Y$, Chiguchi M, Ishibashi T, Aso T. Soy intake related to menopausal symptoms, serum lipids, and bone mineral density in postmenopausal Japanese women. Obstet Gynecol. 2001;97:109-15. 
27. González CA. Estimation of dietary sources and flavonoid intake in a Spanish adult population (EPIC-Spain). J Am Diet Assoc. 2010;110:390-8.

28. Larkin T, Price WE, Astheimer L. The key importance of soy isoflavone bioavailability to understanding health benefits. Crit Rev Food Sci Nutr. 2008;48:538-52.

29. Vincent A, Lorraine A, Fitzpatrick MD. Soy isoflavones: are they useful in menopause? Mayo Clin Proc. 2000;75:1174-84.

30. Gold E, Sternfeld B, Kelsey J, et al. Relation of demographic and lifestyle factors to symptoms in a multi-racial/ethnic population of women 40-55 years of age. Am J Epidemiol. 2000;152:463-73.

31. Cornwell T, Cohick W, Raskin I. Dietary phytoestrogens and health. Phytochemistry. 2004;656:995-1016.

32. Kurzer M. Phytoestrogen supplement use by women. J Nutr. 2003;133:19835-65.

33. Bhagwat S, Haytowitz DB, Holden JM. USDA Database for the Isoflavone Content of Selected Foods. Beltsville: Human Nutrition Research Center, U.S. Department of Agriculture; 2008.

34. Vergne S, Titier K, Bernard V, et al. Bioavailability and urinary excretion of isoflavones in humans: effects of soy-based supplements formulation and equol production. J Pharm Biomed Anal. 2007;43:1488-94.

35. Nielsen IL, Williamson G. Review of the factors affecting bioavailability of soy isoflavones in humans. Nutr Cancer. 2007;57:1-10.

36. Cassidy A, Albertazzi P, Lise Nielsen I, et al. Critical review of health effects of soyabean phyto-oestrogens in postmenopausal women. Proc Nutr Soc. 2006;65:76-92.

37. Taku K, Lin N, Cai D, et al. Effects of soy isoflavone extract supplements on blood pressure in adult humans: systematic review and meta-analysis of randomized placebo-controlled trials. J Hypertens. 2010;28:1971-82.

38. Li SH, Liu XX, Bai YY, et al. Effect of oral isoflavone supplementation on vascular endothelial function in postmenopausal women: a meta-analysis of randomized placebo-controlled trials. Am J Clin Nutr. 2010;91:480-6.

39. Taku K, Umegaki K, Sato Y, et al. Soy isoflavones lower serum total and LDL cholesterol in humans: a meta-analysis of 11 randomized controlled trials. Am J Clin Nutr. 2007;85: 1148-56.

40. Zhuo XG, Melby MK, Watanabe S. Soy isoflavone intake lowers serum LDL cholesterol: a meta-analysis of 8 randomized controlled trials in humans. J Nutr. 2004;134:2395-400.

41. Weggemans RM, Trautwein EA. Relation between soy-associated isoflavones and LDL and HDL cholesterol concentrations in humans: a meta-analysis. Eur J Clin Nutr. 2003;57:940-6.

42. Weggemans R, Trautwein EA. Relation between soy-associated isoflavons and LDL and $\mathrm{HDL}$ colesterol concentrations in humans: a metanalysis. Eur J Cli Nutr. 2003;57:940-6.

43. European Food Safety Authority (EFSA). Scientific Opinion on the substantiation of a health claim related to soy protein and reduction of blood cholesterol concentrations pursuant to Article 14 of the Regulation (EC) No 1924/2006. EFSA J. 2010;8:1688.

44. Bolaños R, Francia J. Isoflavones versus hormone therapy for reduction of vertebral fracture risk: indirect comparison. Menopause. 2010;17:1201-5.

45. Taku K, Melby MK, Kurzer MS, et al. Effects of soy isoflavone supplements on bone turnover markers in menopausal women: systematic review and meta-analysis of randomized controlled trials. Bone. 2010;47:413-23.

46. Taku K, Melby MK, Takebayashi J, et al. Effect of soy isoflavone extract supplements on bone mineral density in menopausal women: meta-analysis of randomized controlled trials. Asia Pac J Clin Nutr. 2010;19:33-42.
47. Liu J, Ho SC, Su YX, et al. Effect of long-term intervention of soy isoflavones on bone mineral density in women: a metaanalysis of randomized controlled trials. Bone. 2009;44: 948-53.

48. Ma DF, Qin LQ, Wang PY, Katoh R. Soy isoflavone intake increases bone mineral density in the spine of menopausal women: meta-analysis of randomized controlled trials. Clin Nutr. 2008;27:57-64.

49. Ma DF, Qin LQ, Wang PY, Katoh R. Soy isoflavone intake inhibits bone resorption and stimulates bone formation in menopausal women: meta-analysis of randomized controlled trials. Eur J Clin Nutr. 2008;62:155-61.

50. Koh WP, Wu AH, Wang R, et al. Gender-specific associations between soy and risk of hip fracture in the Singapore Chinese Health Study. Am J Epidemiol. 2009;170:901-9.

51. European Food Safety Authority (EFSA). Scientific Opinion on the substantiation of health claims related to soy isoflavones and maintenance of bone mineral density (ID 1655) pursuant to Article 13(1) of Regulation (EC) No 1924/20061. EFSA J. 2009;7:1270.

52. Bolaños R, Del Castillo A, Francia J. Soy isoflavones versus placebo in the treatment of climacteric vasomotor symptoms: systematic review and meta-analysis. Menopause. 2010;17:660-6.

53. Lethaby AE, Brown J, Marjoribanks J, Kronenberg F, Roberts H, Eden J. Phytoestrogens for vasomotor menopausal symptoms. Cochrane Database Syst Rev. 2007;(4):CD001395.

54. Howes LG, Howes JB, Knight DC. Isoflavone therapy for menopausal flushes: a systematic review and meta-analysis. Maturitas. 2006;55:203-11.

55. Nelson HD, Vesco KK, Haney E, et al. Nonhormonal therapies for menopausal hot flashes: systematic review and metaanalysis. JAMA. 2006;295:2057-71.

56. Durán M. Fitoestrógenos. Rev Gin Obst. 2001;2:138-47.

57. European Food Safety Authority (EFSA). Scientific Opinion on the substantiation of health claims related to soy isoflavones and protection of DNA, proteins and lipids from oxidative damage, maintenance of normal blood LDL-cholesterol concentrations, reduction of vasomotor symptoms associated with menopause, maintenance of normal skin tonicity, contribution to normal hair growth, "cardiovascular health", treatment of prostate cancer and "upper respiratory tract" pursuant to Article 13(1) of Regulation (EC) No 1924/20061. EFSA J. 2011;9:2264.

58. Dong JY, Qin LQ. Soy isoflavones consumption and risk of breast cancer incidence or recurrence: a meta-analysis of prospective studies. Breast Cancer Res Treat. 2011;125:315-23.

59. Enderlin CA, Coleman EA, Stewart CB, Hakkak R. Dietary soy intake and breast cancer risk. Oncol Nurs Forum. 2009;36: 531-9.

60. Hooper L, Madhavan G, Tice JA, et al. Effects of isoflavones on breast density in pre- and post-menopausal women: a systematic review and meta-analysis of randomized controlled trials. Hum Reprod Update. 2010;16:745-60.

61. Qin LQ, Xu JY, Wang PY, Hoshi K. Soyfood intake in the prevention of breast cancer risk in women: a meta-analysis of observational epidemiological studies. J Nutr Sci Vitaminol (Tokyo). 2006;52:428-36.

62. Shimazu T, Inoue M, Sasazuki SJ, et al. PHC Study Group. Plasma isoflavones and the risk of lung cancer in women: a nested case-control study in Japan. Cancer Epidemiol Biomarkers Prev. 2011;20:419-27.

63. Shimazu $T$, Inoue $M$, et al. Isoflavone intake and risk of lung cancer: a prospective cohort study in Japan. Am J Clin Nutr. 2010;91:722-8.

64. Travis RC, Allen NE, et al. A prospective study of vegetarianism and isoflavone intake in relation to breast cancer risk in British women. Int J Cancer. 2008;122:705-10. 
65. Kurahashi N, Inoue M, et al; JPHC Study Group. Isoflavone consumption and subsequent risk of hepatocellular carcinoma in a population-based prospective cohort of Japanese men and women. Int J Cancer. 2009;124:1644-966.

66. Kurahashi $N$, Iwasaki $M$, et al. Isoflavones and subsequent risk of prostate cancer in a nested case-control study: the Japan Public Health Center. J Clin Oncol. 2008;26:5923-9.

67. Akhter $M$, Inoue $M$, Kurahashi $N$, et al. Japan Public Health Center-Based Prospective Study Group. Dietary soy and isoflavone intake and risk of colorectal cancer in the Japan public health center-based prospective study. Cancer Epidemiol Biomarkers Prev. 2008;17:2128-35.

68. Wu AH, Koh WP, Wang R, Lee HP. Soy intake and breast cancer risk in Singapore Chinese Health Study. Br J Cancer. 2008;99:196200.
69. Iwasaki M, Inoue M, Otani T, et al; Japan Public Health Centerbased prospective study group. Plasma isoflavone level and subsequent risk of breast cancer among Japanese women: a nested case-control study from the Japan Public Health Center-based prospective study group. J Clin Oncol. 2008;26: 1677-83.

70. Guha N, Kwan ML, et al. Soy isoflavones and risk of cancer recurrence in a cohort of breast cancer survivors: the Life After Cancer Epidemiology study. Breast Cancer Res Treat. 2009;118:395-405.

71. Park SY, Murphy SP, Wilkens LR, et al. Legume and isoflavone intake and prostate cancer risk: The Multiethnic Cohort Study. Int J Cancer. 2008;123:927-32.

72. Mathew BC, Reji SD, Jamal AB, Campbell IW. Phytoestrogens and cancer. J Inst Med. 2009;21:11-9. 